\title{
sFas and sFas Ligand and Pediatric Sepsis-Induced Multiple Organ Failure Syndrome
}

\author{
LESLEY DOUGHTY, ROBERT S.B. CLARK, SANDRA S. KAPLAN, HOWELL SASSER, AND \\ JOSEPH CARCILLO \\ Department of Pediatrics, Rhode Island Hospital, Providence, Rhode Island 02903, U.S.A. [L.D.]; \\ Departments of Anesthesiology/CCM [R.S.B.C., J.C.], Pediatrics [R.S.B.C., J.C.], and Pathology [S.S.K.], \\ Center for Clinical Pharmacology [J.C.], and School of Public Health [H.S.], University of Pittsburgh,
} Pittsburgh, Pennsylvania 15261, U.S.A.

\begin{abstract}
The Fas-Fas ligand system is important for apoptosis of activated immune cells. Perturbation of this system occurs in diseases with dysregulated inflammation. Increased soluble Fas (sFas) occurs in systemic inflammatory response syndrome (SIRS) and can block apoptosis. Increased shedding of FasL (sFasL) occurs in viral infection and hepatitis. Although dysregulated inflammation is associated with sepsis-induced multiple organ failure (MOF) in children, a role for Fas has not been established. We hypothesize that 1) sFas will be increased in children with severe and persistent sepsis-induced MOF and will correlate with inflammatory markers suggesting a role for sFas in inflammatory dysregulation in severe sepsis, and 2) sFasL will be increased when viral sepsis or sepsis-induced liver failureassociated MOF is present in children. Plasma sFas, sFasL, IL-6, IL-10, nitrite + nitrates, and organ failure scores were measured on $\mathrm{d} 1$ and $\mathrm{d} 3$ in 92 children with severe sepsis and 12 critically ill control children. sFas levels were increased in severe sepsis, continued to increase in persistent MOF and nonsurvivors, and were correlated with serum inflammatory markers (IL-6, IL-10, nitrite + nitrate levels). In contrast, sFasL was not increased in severe sepsis and did not correlate with inflammation. sFasL was, however, increased in liver failure-associated MOF and in nonsurvivors, and was associated with viral infection. At autopsy, hepatocyte destruction and lymphocyte infiltration were associ-
\end{abstract}

\section{ABSTRACT}

ated with increased sFas and sFasL levels. sFas may interfere with activated immune cell death and contribute to dysregulation of inflammation, worsening outcome from severe sepsis. sFasL may contribute to hepatic injury and the development of liver failure-associated MOF. (Pediatr Res 52: 922-927, 2002)

$\quad$ Abbreviations
MAP, mean arterial pressure
MOF, multiple organ failure
OFI, organ failure index
DIC, disseminated intravascular coagulation
PMOF, persistent multiple organ failure
SFas, soluble Fas
SFasL, soluble Fas ligand
FasL, Fas ligand
NMOF, no multiple organ failure
NK, natural killer cells
SMOF, sequential multiple organ failure
SLE, systemic lupus erythematosus
TNF, tumor necrosis factor
AICD, activated immune cell death
EBV, Epstein-Barr virus
SIRS, systemic inflammatory response syndrome

Regulation of the inflammatory response is complex and poorly understood. AICD via apoptosis is a mechanism thought to be important in regulating proliferating immune cells. Apoptosis has been observed in each immune cell type after activation and may be important in terminating the immune response (1-3). The Fas-FasL system is recognized as a major pathway for the induction of apoptosis in activated immune cells (1-3). Fas (CD 95) is a type I membrane protein

Received December 18, 2000; accepted July 3, 2002.

Correspondence: Lesley Doughty, M.D., Rhode Island Hospital, 593 Eddy Street, Potter 112, Providence, RI 02903, U.S.A.

Supported in part by MM301GCRC.

DOI: 10.1203/01.PDR.0000036279.41965.F7 of the TNF-receptor family of proteins and is widely expressed in many tissue types, both constitutively and after activation (4, 5). Expression of FasL, a type II membrane protein with significant homology to TNF- $\alpha$, is restricted to activated $\mathrm{T}$ and B lymphocytes and NK cells (5). The interaction of FasL with Fas on a target cell triggers apoptosis of that cell (4). Because the expression of FasL is regulated more strictly than Fas, its expression by effector cells is thought to be a triggering event in inducing programmed cell death (6).

Fas-mediated apoptosis is suppressed by incubating leukocytes in vitro with proinflammatory cytokines and endotoxin $(7,8)$. Several hypotheses have been suggested to explain this including release of a soluble form of Fas capable of blocking 
the interaction of membrane-bound Fas with its ligand, thereby preventing apoptosis $(3,9)$. The addition of sFas perpetuates proliferation of stimulated $\mathrm{T}$ cells in culture (10). Several studies have shown reduced Fas-mediated apoptosis of neutrophils obtained from humans suffering from the systemic inflammatory response syndrome, burn injuries, or surgical trauma $(11,12)$. The shedding of sFas may represent a controlled host response to inflammation allowing activated immune cell survival until infection or tissue injury is resolved.

Interruption of Fas-mediated apoptosis has been implicated in disease states characterized by dysregulated inflammation. Mutations in both the Fas receptor (lpr/lpr) or the FasL ( $g l d /$ $g l d)$ are present in mouse models of SLE in which accumulation of lymphocytes occurs, resulting in pathologic condition (4). Administration of exogenous sFas can induce autoimmune disease experimentally, and sFas serum levels are increased in human SLE suggesting that sFas can block apoptosis resulting in clinically significant pathophysiology (9).

Perturbation of Fas-FasL-mediated AICD may also contribute to the pathogenesis of sepsis-induced MOF. A potentiated inflammatory response to bacterial superantigen is seen in the lpr/lpr mice suggesting that Fas signal disruption may permit a more vigorous inflammatory response (13). A dysregulated proinflammatory response has been hypothesized as an explanation for the development of sepsis-induced MOF. In children with persistent sepsis-induced MOF, uncontrolled inflammation exists as indicated by increased markers of both the proand antiinflammatory response (serum IL-6, IL-10, and nitrite + nitrate levels) (14-16). Adults with MOF demonstrate higher sFas serum levels, which correlate with serum nitrite + nitrate levels, supporting this hypothesis $(17,18)$. A role for sFas and failed AICD has yet to be evaluated in children with sepsis and MOF.

Shedding of the sFasL may contribute to the development of sepsis-induced MOF through another mechanism. Viral infection activates metalloproteinases on the surface of lymphocytes and NK cells causing shedding of FasL (5). sFasL as well as agonist Fas antibody (mimcs sFasL) are capable of inducing cytotoxicity, hepatocyte destruction, and mortality in mice through the interaction with hepatocyte Fas (19-23). Morerecent data have demonstrated that membrane-bound FasL is much more efficient at killing Fas-bearing cells. In fact, sFasL administered at low doses inhibits hepatocyte cytotoxicity by occupying but not inducing the cross-linking of membranebound Fas receptors necessary for Fas signaling (19-22). In human disease, however, serum sFasL levels are increased in leukemias and lymphomas presenting with remote organ injury caused by lymphoproliferation as well as viral hepatitis. SFasL levels correlate with organ injury severity scores suggesting that sFasL may in fact be cytotoxic in disease states characterized by increased expression and shedding of $\mathrm{sFasL}$ despite conflicting data from animal and immortalized cell line studies (24-26).

Children with severe sepsis commonly develop MOF. Unlike adults, the majority of these children (90\%) present with a simultaneous pattern of organ failure. A smaller group develops MOF in a sequential manner that is more typical of the adult pattern of MOF $(17,18)$. SMOF syndrome is character- ized by respiratory failure followed by the development of liver and renal failure. Because activation of Fas signaling is associated with liver injury, it is plausible that sFasL is involved in the pathogenesis of liver failure-dependent SMOF in children.

In the present study we tested two hypotheses in children with severe sepsis syndrome. First, if perpetuation of activated immune cells plays a role in PMOF through Fas-dependent mechanisms, then exaggerated inflammation should be associated with increased sFas shedding over time. Second, if sFasL is shed by viral activation of immune cells and if it is deleterious to hepatocyte function, then increased sFasL levels should be associated with viral sepsis, liver dysfunction, and the development of SMOF.

\section{METHODS}

Patient selection. The Children's Hospital of Pittsburgh Institutional Review Board approval was obtained for this protocol, and informed consent was obtained. Consecutive children with a presumed diagnosis of sepsis were enrolled within $24 \mathrm{~h}$ of presentation to the pediatric intensive care unit. The criteria for sepsis used were modifications of those described by Bone et al. (27) including clinical suspicion of sepsis, hyper- or hypothermia (rectal temperature $>38.5^{\circ}$ or $<35^{\circ} \mathrm{C}$ ), tachycardia, and at least one of the following indications of altered organ perfusion and function: altered mental status, hypoxemia, elevated serum lactate level, oliguria, delayed capillary refill $(>5 \mathrm{~s})$, or bounding pulses. Exclusion criteria included death within the first $2 \mathrm{~d}$ of the study. Children receiving any exogenous nitric oxide source such as inhaled nitric oxide, nitroprusside, or nitroglycerin were excluded from analyses involving nitrite + nitrate levels. Fourteen critically ill children who were afebrile and not suspected of having infection were sampled on d 1 and used as a control group.

Each child was assigned an OFI score daily for the first $3 \mathrm{~d}$. The components of the OFI are modifications of the multiple organ system failure scoring system for children described by Wilkinson et al. (28) and Proulx et al. (29) (Table 1). Children were grouped on the basis of the OFI. Children were separated into PMOF, which was 3 OFI on d 3, resolved MOF, in which 3 OFI present on $\mathrm{d} 1$ or 2 improved to $<3$ by $\mathrm{d} 3$, and NMOF, in which $<3$ OFI was present throughout the 3 -d course (15, 16). In a separate analysis children were grouped according to the pattern of MOF developed. Any child who developed hepatic and renal failure after presenting with respiratory failure was designated as SMOF and all others were designated no SMOF $(15,16)$. Nonsurvivors were those children who survived the initial 3-d sampling and scoring period but did not survive beyond $14 \mathrm{~d}$.

sFas, sFasL, and cytokine plasma levels. Two milliliters of blood was obtained on $\mathrm{d} 1$ and 3 of sepsis. sFas, sFasL, IL-10, and IL-6 plasma levels were quantified by ELISA with reagents and kits from Oncogene Research Products (Cambridge, MA, U.S.A.), Medical and Biologic Laboratories Co, LTD (Nagova, Japan), Pharmingen (San Diego, CA, U.S.A.), and Genzyme (Cambridge, MA, U.S.A.), respectively.

Nitrite + nitrate determination. The plasma samples were deproteinized followed by direct measurement of plasma nitrite 
Table 1. Organ failure index*

\begin{tabular}{|c|c|}
\hline Cardiovascular & $\begin{array}{l}\text { MAP }<5 \text { th percentile for age, or } \\
\text { requirement for vasopressor } \\
\text { agents after volume resuscitation }\end{array}$ \\
\hline Pulmonary & $\begin{array}{l}\mathrm{PaO}_{2} / \mathrm{FiO}_{2} \text { ratio }<300 \text { and ventilator } \\
\text { requirement }\end{array}$ \\
\hline Renal & $\begin{array}{l}\text { Oliguria }<1 \mathrm{~mL} / \mathrm{kg} / \mathrm{h} \text { average for } \\
\geq 8 \mathrm{~h} \text { if }<30 \mathrm{~kg},<0.5 \mathrm{~mL} / \mathrm{kg} / \mathrm{h} \\
\text { average for } 8 \mathrm{~h} \text { if }>30 \mathrm{~kg} \text {, or } \\
\text { creatinine }>1.0\end{array}$ \\
\hline Hematologic & $\begin{array}{l}\mathrm{PT}, \mathrm{PTT}>1.5 \times \text { normal and } \\
\quad \text { platelets }<100,000\end{array}$ \\
\hline Hepatic & $\begin{array}{l}\text { ALT, AST }>100 \text { and total bilirubin } \\
>1.0 \text { (excluding neonates) }\end{array}$ \\
\hline $\mathrm{CNS}$ & $\begin{array}{l}\text { Glasgow Coma Scale }<12 \text { (prior to } \\
\text { sedation) }\end{array}$ \\
\hline
\end{tabular}

Maximum score $=\overline{6}$

* Organ failure is defined by meeting all criteria for each organ system. The most abnormal values of the day were used to determine the daily score. The organ failure index is the sum of points given for each type of organ failure. Elevated bilirubin in neonates was not scored.

Abbreviations: MAP, mean arterial pressure; $\mathrm{PaO}_{2}$, arterial oxygen pressure; $\mathrm{FiO}_{2}$, fraction of inspired oxygen; PT, pro-thrombin time; PTT, partial thromboplastin time; ALT, alanine transaminase; AST, aspartate transaminase.

levels. Also, the plasma nitrate was reduced (by cadmium reduction) to nitrite with subsequent nitrite quantitation. Nitrite levels were quantitated using the Griess reaction (30). Data are reported as nitrite plus nitrate values in moles per liter.

Statistics. Two-way repeated measures ANOVA with Student-Newman-Keuls test for multiple comparisons was used to compare sFas and sFasL levels in children with PMOF, resolved MOF, or NMOF; SMOF versus NMOF, and nonsurvivors versus survivors. Mann-Whitney rank sum was used to compare all children with sepsis to control children. Generalized estimating equations were used to examine relationships between Fas and FasL with the presence of organ failure, bacterial, fungal, and viral culture status, age, and transplant status. Spearman correlation, $\rho$, was used to determine associations between cytokine levels and Fas and FasL levels. Statistics were evaluated using SAS Institute (Cary, NC, U.S.A.).

\section{RESULTS}

Ninety-two children with sepsis were enrolled, with ages ranging from $1 \mathrm{~d}$ to $20 \mathrm{y}$. The cause of sepsis was $56 \%$ bacterial, (50\% being Gram-positive and 50\% Gram-negative), $12 \%$ viral, $8 \%$ fungal, and $22 \%$ culture-negative. Thirty-five percent had no other medical problems, $22 \%$ were transplant recipients (four bone marrow, 18 solid organ), 14\% had malignancies, $13 \%$ were newborns, $7 \%$ had cerebral palsy or developmental delay, $3 \%$ were awaiting transplants, $2 \%$ were postoperative, $1 \%$ had trauma, and $1 \%$ had a known immune deficiency syndrome. Twenty-two percent were neutropenic. Eight children had SMOF. Four children had lymphoproliferative disease, two were primary EBV-related, and two were posttransplantation EBV-related. Plasma samples were obtained from 12 critically ill control patients. Their diagnoses included status asthmaticus, status epilepticus, severe closed head injury, gunshot wound, multiple trauma, end-stage liver disease, ventriculoperitoneal shunt malfunction, tricyclic antidepressant overdose, CNS resection, and persistent pulmonary hypertension.

sFas levels were increased in sepsis patients on d 3 only compared with control patients and in nonsurvivors compared with survivors $(p<0.01$; Table 2$)$. Increasing sFas levels $\mathrm{s}$ a function of time were present in children with PMOF $(p<$ $0.01)$ and SMOF $(p<0.001)$, and tended to be increased in nonsurvivors $(p<0.06$; Table 2$)$. Increased sFas levels were associated with fungal infection $(p<0.05)$ and lymphoproliferative disease $(p<0.001)$. Multivariate analysis controlling for age, transplant status, and infection cause showed an association between sFas and hematologic $(p<0.001)$ and hepatic $(p<0.001)$ failure. sFas levels correlated to serum IL-6, IL-10, nitrite + nitrate levels, and OFI on d 3 ( $\rho=$ $0.3-0.5 ; p<0.05)$.

There was no difference found between sFasL levels in the sepsis and control population; however, increased sFasL levels were present in children with SMOF $(p<0.01)$ and nonsurvivors $(p<0.05$; Table 3$)$. Increased $\mathrm{sFasL}$ was associated with viral infection $(p<0.005)$ and lymphoproliferative disease $(p<0.0001)$. To exclude the possibility that the associ-

Table 2. Plasma levels of sFas in critically ill control and sepsis patients

\begin{tabular}{lcc}
\hline & \multicolumn{1}{c}{ sFas $(\mathrm{U} / \mathrm{mL})$ mean \pm SD; median $[\mathrm{min}-\mathrm{max}]$} \\
\cline { 2 - 3 } Group & \multicolumn{1}{c}{ Day 1} & Day 3 \\
\hline Control $(n=12)$ & $3.4 \pm 1.4 ; 3.2[1.9-6.3]$ & - \\
Sepsis $(n=92)$ & $6.6 \pm 6.3 ; 4.4[0.2-4]$ & $8.4 \pm 8.2 ; 5.2[0.7-39.8]^{*}$ \\
PMOF $(n=26)$ & $8.1 \pm 7.0 ; 5.0[0.2-21.5]$ & $13.1 \pm 11.5 ; 10.1[0.7-39.8] \dagger$ \\
RMOF $(n=28)$ & $7.5 \pm 7.6 ; 4.5[2.1-37]$ & $8.6 \pm 6.4 ; 6.6[1.6-31.5]$ \\
NMOF $(n=38)$ & $4.8 \pm 4.3 ; 3.2[0.7-19.6]$ & $5.1 \pm 4.4 ; 3.6[0.7-24.7]$ \\
SMOF $(n=8)$ & $11.6 \pm 8.2 ; 13.4[1.7-21.5]$ & $19.4 \pm 12.9 ; 16.5[3.4-39.8] \ddagger$ \\
No SMOF $(n=84)$ & $6.1 \pm 6.0 ; 4.3[0.2-37]$ & $7.4 \pm 6.9 ; 5.2[0.7-38.7]$ \\
Nonsurvivors $(n=14)$ & $9.4 \pm 7.0 ; 6.4[1.6-21.5] \S$ & $15.1 \pm 11 ; 12.1[2.7-38.3] \S$ \\
Survivors $(n=78)$ & $6.1 \pm 6.1 ; 4.1[0.2-37]$ & $7.2 \pm 7.0 ; 5[0.7-39.8]$ \\
\hline
\end{tabular}

Abbreviation: RMOF, resolved MOF.

$* p<0.05$ increased sFas $v s$ control (Mann-Whitney rank sum test).

$\dagger p<0.05$ increased sFas over time vs RMOF and NMOF (2-way repeated measures ANOVA).

$\ddagger p<0.05$ increased sFas over time $v s$ no sequential MOF (2-way repeated measures ANOVA).

$\S p<0.05$ increased sFas $v s$ survivors (2-way repeated measures ANOVA). 
Table 3. Plasma levels of sFasL in sepsis and control patients

\begin{tabular}{llc}
\hline & \multicolumn{1}{c}{ SFas L $(\mathrm{pg} / \mathrm{mL})$ mean \pm SD; median $[\mathrm{min}-\mathrm{max}]$} \\
\cline { 2 - 3 } Group & \multicolumn{1}{c}{ Day 1} & Day 3 \\
\hline Control $(n=12)$ & $171 \pm 132 ; 150[0-500]$ & - \\
Sepsis $(n=92)$ & $262 \pm 378 ; 160[0-2400]$ & $250 \pm 445 ; 110[0-3600]$ \\
PMOF $(n=26)$ & $358 \pm 545 ; 180[0-2400]$ & $381 \pm 735 ; 160[0-3600]$ \\
RMOF $(n=28)$ & $251 \pm 340 ; 125[0-1600]$ & $186 \pm 260 ; 100[0-1000]$ \\
NMOF $(n=38)$ & $197 \pm 218 ; 105[0-1004]$ & $771 \pm 1256 ; 230[0-3600] *$ \\
SMOF $(n=8)$ & $666 \pm 901 ; 225[0-2400]^{*}$ & $198 \pm 229 ; 100[0-1002]$ \\
No SMOF $(n=84)$ & $222 \pm 264 ; 140[0-1600]$ & $533 \pm 1015 ; 150[0-3600] \dagger$ \\
Nonsurvivors $(n=14)$ & $473 \pm 694 ; 160[0-2400] \dagger$ & $201 \pm 232 ; 100[0-1002]$ \\
Survivors $(n=78)$ & $219 \pm 265 ; 140[0-1600]$ & \\
\hline
\end{tabular}

$* p<0.05$ increased sFasL vs NSMOF.

$\dagger p<0.05$ increased sFasL $v$ s survivors.

ation between sFasL levels and SMOF was solely a result of children with lymphoproliferative or viral disease $(25 \%$ of SMOF and $11 \%$ no SMOF, $p=$ NS), these children were excluded and the analysis was repeated, still demonstrating increased sFasL levels in children with SMOF $(p<0.008)$. Multivariate analysis controlling for age, transplant status, and infection cause showed an association between $\mathrm{sFas} \mathrm{L}$ and hematologic $(p<0.005)$ and hepatic $(p<0.005)$ failure. sFasL did not correlate with serum IL-6, IL-10, nitrite + nitrate levels, or OFI.

Twelve of 14 nonsurvivors underwent autopsy (Table 4). Hepatic lymphocyte infiltration was present in six of the seven patients with sFasL levels greater than $150 \mathrm{ng} / \mathrm{mL}$ (median for control patients) on $\mathrm{d} 3$ of sepsis. Three of the four patients with the highest sFasL levels had variable degrees of hepatic injury, and the child with the highest sFas and sFasL levels showed massive liver destruction. The five children with $\mathrm{sFasL}$ levels below $150 \mathrm{ng} / \mathrm{mL}$ on $\mathrm{d} 3$ showed no evidence of hepatic lymphocyte infiltration or injury.

\section{DISCUSSION}

Our study supports a role for the Fas-FasL system in the development of sepsis-induced MOF. Children with PMOF and nonsurvivors, who have a dysregulated inflammatory re- sponse, displayed increasing serum sFas levels with time. Nonsurvivors and children who developed the sequential form of MOF involving the liver had increased levels of both $\mathrm{sFas}$ and sFasL, suggesting that organ injury, particularly hepatic, may be Fas-related. Either sFas-mediated inhibition of AICD perpetuating inflammation or FasL-mediated organ injury from infiltrating lymphocytes shedding or bearing sFasL could explain this observation. This concept is supported by our autopsy findings, which demonstrate an association between hepatic lymphocytic infiltration, increased sFasL levels, and severe liver destruction.

The source of $\mathrm{sFas}$ in sepsis-induced MOF is not known. Increased $\mathrm{sFas}$ has been reported in lymphomas (not myelodysplasias), thrombotic thrombocytopenic purpura, DIC, SLE with organ injury (hepatic and renal), and MOF (31-34), suggesting activated lymphocytes or possibly endothelium as sources for increased shedding of sFas. Others report that serum sFas levels reflect the severity of hepatic injury and hepatic Fas expression in fulminant and chronic hepatitis (25, $35)$. We also observed increased serum sFas in children with sepsis compared with control children and noted a link between increased sFas levels and liver or hematologic failure (DIC). It is not known whether the source of sFas in sepsis-induced MOF is related to shedding from inflammatory cells, injured

Table 4. Pathologic diagnosis, sFas and sFasL levels $(d 1, d 3)$, and liver pathology in 12 children in whom autopsies were performed

\begin{tabular}{|c|c|c|c|c|c|}
\hline \multirow[b]{2}{*}{ Diagnosis } & \multicolumn{2}{|c|}{ sFas $(\mathrm{U} / \mathrm{mL})$} & \multicolumn{2}{|c|}{ sFasL (pg/mL) } & \multirow[b]{2}{*}{ Liver pathology } \\
\hline & Day 1 & Day 3 & Day 1 & Day 3 & \\
\hline LPD, Candida & 17 & 20 & 2400 & 3600 & $\begin{array}{l}\text { Massive liver necrosis, } \\
\text { LPD, EBV+ }\end{array}$ \\
\hline Histiocytosis, Candida & 22 & 17 & 1700 & 1600 & $\begin{array}{l}\text { Liver necrosis, chronic } \\
\text { inflammation }\end{array}$ \\
\hline OLTx, Enterobacter cloacae & 5 & 4 & 720 & 600 & $\begin{array}{l}\text { Liver necrosis, } \\
\text { Lymphocyte infiltration }\end{array}$ \\
\hline OLTx, LPD & 21 & 39 & 240 & 222 & LPD, EBV+ \\
\hline Lymphangiectasia & 5 & 8 & 100 & 240 & Micronodular cirrhosis \\
\hline OLTx, LPD & 7 & 13 & 20 & 240 & LPD, EBV+ \\
\hline ARDS & 5 & 3 & 8 & 140 & Normal \\
\hline ALL, Candida & 5 & 10 & 48 & 48 & Candida \\
\hline OLTx & 4 & 12 & 0 & 0 & Congestion \\
\hline
\end{tabular}

LPD, lymphoproliferative disease; OLTx, orthotopic liver transplant; ARDS, adult respiratory distress syndrome; ALL, acute lymphocytic leukemia; Ht, heart; Tx, transplant. 
endothelium, or injured parenchyma. No correlation between $\mathrm{sFas}$ (or sFasL) and renal failure exists to suggest that clearance of these molecules was impaired.

Increased $\mathrm{sFas}$ shedding was associated with increased levels of inflammatory mediators. Like inflammatory cytokines and nitric oxide, increased sFas levels correlated with a worsening OFI, suggesting a link between continued sFas shedding, dysregulated inflammation, and the development of MOF. sFas levels continued to increase with time in PMOF and SMOF, supporting sFas as a potential effector in sepsis-induced MOF syndromes. We speculate that because increasing sFas levels were associated with infections not rapidly responsive to therapy, namely fungal and EBV-related lymphoproliferative disease, persistent infection or antigen presence may perpetuate the inflammatory response via release of sFas.

Experimental administration of agonistic antibody to Fas, which has sFasL-like activity, induces fulminant liver destruction and lethality via hepatocyte Fas interaction, leading to activation of caspases central to many apoptotic pathways (23, $26,36)$. Lethality and liver damage induced by this treatment can be abrogated by apoptosis inhibitors (36-39). These studies indicate that activation of hepatocyte Fas via sFasL-like molecules is an important mechanism for liver injury.

Many experimental studies support a pathologic role for FasL in animal models of hepatitis, endotoxemia, and polymicrobial sepsis, as well as graft-versus-host disease $(22,26,40$, 41). In human disease, increased circulating sFasL was found in aggressive lymphomas presenting with distant organ failure, hepatitis, and hemophagocytic lymphocytic histiocytosis and Diamond-Blackfan syndrome $(24,42)$. Experimental administration of recombinant $\mathrm{sFasL}$ induced cell death in vitro and lethality, which was potentiated by preexisting inflammation (22). These findings support sFasL as a trigger for cell death with its deleterious effect potentially greatest in the setting of systemic inflammation. Some reports suggest that FasL must be membrane-bound to induce apoptosis via receptor crosslinking on Fas-bearing cells. These reports also suggest that sFasL without receptor cross-linking ability may, like sFas, inhibit Fas signal transduction; however, the potentiating role for preexisting inflammation was not assessed in these models (19-21). Currently, a complete understanding of the role of this molecule in human disease is not well defined, and existing data do not rule out the possibility that tissue specificities may determine the differential effects of sFasL from membranebound FasL during inflammatory processes.

In our study, sFasL levels were associated with viral sepsis and EBV-induced lymphoproliferative disease. T cells and NK cells can release sFasL when activated, and increased serum sFasL levels in viral disease may represent lymphocyte activation critical to eradication of virus-infected cells. In contrast to sFas, sFasL levels did not correlate with markers of inflammation or development of PMOF. This finding suggests that sFasL may have a different role compared with sFas in the children with severe septic shock. In agreement with adult studies, both sFasL and sFas levels were associated with liver failure and DIC (22-26, 33-35). Current literature suggests that $\mathrm{sFasL}$ may interact with hepatic Fas and induce liver injury, thereby inducing shedding of sFas $(24,25,36)$. We extended these findings by demonstrating in children an association between sFas and sFasL with sepsis-induced liver failure and with SMOF, a liver failure-dependent categorization. FasL on infiltrating lymphocytes may also interact with hepatocyte Fas independent of the effect of circulating sFasL $(25,34)$. Our autopsy results support a role for Fas in sepsisinduced liver injury, possibly mediated by hepatocyte lymphocyte infiltration. The seven nonsurvivors with hepatic lymphocyte infiltration and hepatic destruction had higher sFas and sFasL levels than the five nonsurvivors without hepatic lymphocyte infiltration or injury.

Our study is limited by an inability to determine the source of circulating sFas and sFasL as well as an inability to establish any cause and effect relationships. Unlike previous studies in adult patients, we did not perform sequential flow cytometry evaluations of circulating inflammatory cell populations to determine whether sFas prevented AICD, and we did not obtain liver biopsies to establish a relationship between $\mathrm{sFas}$ levels, hepatic Fas expression, and apoptosis or sFasL levels and lymphocyte FasL expression. In addition, although sFasL was associated with liver pathologic state and not with inflammatory mediators, we did not determine that $\mathrm{sFasL}$ associations were independent of TNF- $\alpha$, a known mediator of hepatic injury also expressed in septic shock.

\section{CONCLUSIONS}

In conclusion, we have found that $\mathrm{sFas}$ was associated with dysregulated inflammation and PMOF and that increased sFas and $\mathrm{sFasL}$ were associated with liver dysfunction, DIC, and the development of SMOF in children with severe sepsis. Our study suggests that sFasL shedding occurs in severe viral sepsis, and supports a role for perturbed Fas-FasL interactions in lymphoproliferative disease associated with EBV sepsis. Because experimental models suggest that various therapeutic strategies can be effective in Fas-FasL-mediated diseases (23$26,37-42$ ), we speculate that improved understanding of the role of Fas-FasL interactions in humans could have therapeutic implications for children with severe viral sepsis, EBVmediated lymphoproliferative disease, and liver-associated SMOF syndrome.

\section{REFERENCES}

1. Lynch DH, Ramsdell F, Alderson MR 1995 Fas and FasL in the homeostatic regulation of immune responses. Immunol Today 16:569-574

2. Green DR, Scott DW 1994 Activation-induced apoptosis in lymphocytes. Curr Opin Immunol 6:476-487

3. Mountz JD, Zhou T, Wu J, Wang W, Su X, Cheng J 1995 Regulation of apoptosis in immune cells. J Clin Immunol 15:1-16

4. Nagata S, Golstein P 1995 The Fas death factor. Science 267:1449-1456

5. Iwai K, Miyawaki T, Takizawa T, Konno A, Ohta K, Yachie A, Seki H, Taniguchi N 1994 Differential expression of bcl-2 and susceptibility to anti-Fas-mediated cell death in peripheral blood lymphocytes, monocytes, and neutrophils. Blood 84:12011208

6. Liles WC, Kiener PA, Ledbetter JA, Aruffo A, Klebanoff SJ 1996 Differential expression of Fas (CD95) and Fas ligand on normal human phagocytes: implications for the regulation of apoptosis in neutrophils. J Exp Med 184:429-440

7. Kiener PA, Davis PM, Starling GC, Mehlin C, Klebanoff SJ, Ledbetter JA, Liles WC 1997 Differential induction of apoptosis by Fas-Fas ligand interactions in human monocytes and macrophages. J Exp Med 185:1511-1516

8. Mangan DF, Welch GR, Wahl SM 1991 Lipopolysaccharide, tumor necrosis factor-, and IL-1 prevent programmed cell death (apoptosis) in human peripheral blood monocytes. J Immunol 146:1541-1546 
9. Cheng J, Zhou T, Liu C, Shapiro JP, Brauer MJ, Kiefer MC, Barr PJ, Mountz JD 1994 Protection from Fas-mediated apoptosis by a soluble form of the Fas molecule. Science 263:1759-1762

10. Mountz JD, Zhou T, Bluethmann H, Wu J, Edwards 3rd CK 1994 Apoptosis defects analyzed in TcR transgenic and fas transgenic lpr mice. Int Rev Immunol 1:321-342

11. Jimenez MF, Watson RW, Parodo J, Evans D, Foster D, Steinberg M, Rotstein OD Marshall JC 1997 Dysregulated expression of neutrophil apoptosis in the systemic inflammatory response syndrome. Arch Surg 132:1263-1267

12. Chitnis D, Dickerson C, Munster AM, Winchurch RA 1996 Inhibition of apoptosis in polymorphonuclear neutrophils from burn patients. J Leukoc Biol 59:835-839

13. Mountz JD, Baker TJ, Bocherding DR, Bluethmann H, Zhou T, Edwards 3rd CK 1995 Increased susceptibility of fas mutant MRL-1pr/1pr mice to staphylococcal enterotoxin B-induced septic shock. J Immunol 155:4829-4837

14. Doughty LA, Kaplan SS, Carcillo JA 1996 Inflammatory cytokine and nitric oxide responses in pediatric sepsis and organ failure. Crit Care Med 24:1137-1143

15. Doughty LA, Carcillo JA, Kaplan SS, Janosky J 1998 Plasma nitrite and nitrate concentrations and multiple organ failure in pediatric sepsis. Crit Care Med 26:157162

16. Doughty L, Carcillo JA, Kaplan SS, Janosky J 1998 The compensatory antiinflammatory cytokine interleukin 10 response in pediatric sepsis-induced multiple organ failure. Chest 113:1625-1631

17. Endo S, Inada K, Takakuwa T, Kasai T, Yamada Y, Wakabayashi G, Niimi M, Suzuki T, Taniguchi S 1996 Nitrite/nitrate (NOx) and sFas antigen levels in patients with multiple organ failure. Res Commun Mol Pathol Pharmacol 92:253-256

18. Papathanassglou ED, Moynihan JA, Vermillion DL, McDermott MP, Ackerman MH 2000 Soluble Fas levels correlate with multiple organ dysfunction severity, survival, and nitrate levels, but not with cellular apoptotic markers in critically ill patients. Shock 14:107-112

19. Suda T, Hashimoto H, Tanaka M, Ochi T, Nagata S 1997 Membrane Fas ligand kills peripheral blood $\mathrm{T}$ lymphocytes, and soluble fas ligand blocks the killing. J Exp Med $186: 2045-2050$

20. Tanaka M, Itai T, Adachi M, Nagata S 1998 Down regulation of fas ligand by shedding. Nat Med 4:31-36

21. Schneider P, Holler N, Bodmer JL, Hahne M, Frei K, Fontana A, Tschopp J 1998 Conversion of membrane bound fas (CD95) ligand to its soluble form is associated with down regulation of its proapoptotic activity and loss of liver toxicity. J Exp Med 187:1205-1213

22. Tanaka M, Suda T, Yatomi T, Nakamura N, Nagata S 1997 Lethal effects of recombinant human Fas ligand in mice treated with Propionibacterium acnes. J Immunol 158:2303-2309

23. Ogasawara J, Watanabe-Fukunaga R, Adachi M, Matsuzawa A, Kasugai T, Kitamura Y, Itoh N, Suda T, Nagata S 1993 Lethal effect of the anti-fas antibody in mice. Nature 364:806-809

24. Tanaka M, Suda T, Haze K, Nakamura N, Sato K, Kimura F, Motoyoshi K, Mizuki M, Tagawa S, Ohga S, Hatake K, Drummond AH, Nagata 1996 Fas ligand in human serum. Nat Med 2:317-322

25. Ryo K, Kamogawa Y, Yamauchi K, Hayashi N 1996 Involvement of Fas and Fas ligand in case of fulminant hepatitis. Nippon Rinsho 54:1965-1969

26. Kondo T, Suda T, Fukuyama H, Adachi M, Nagata S 1997 Essential roles of the Fas ligand in the development of hepatitis. Nat Med 3:409-413
27. Bone RC, Fischer Jr CJ, Clemmer TP, Slotman GJ, Metz CA, Balk RA 1989 Sepsis syndrome: a valid clinical entity. Crit Care Med 17:389-393

28. Wilkinson JD, Pollack MM, Ruttiman UE, Glass NL, Yeh TS 1986 Outcome of pediatric patients with multiple organ system failure. Crit Care Med 14:271-274

29. Proulx F, Fayon M, Farrell CA, Lacroix J, Gauthier M 1996 Epidemiology of sepsis and multiple organ dysfunction syndrome in children. Chest 109:1033-1037

30. Green LC, Wagner DA, Glogowski J, Skipper PL, Wishnok JS, Tannenbaum SR 1982 Analysis of nitrate, nitrite, and ${ }^{15} \mathrm{~N}$ nitrate in biological fluids. Ann Biochem 126:131-138

31. Niitsu N, Sasaki K, Umeda M 1999 A high serum soluble fas/APO-1 is associated with a poor outcome of aggressive non-Hodgkins lymphoma. Leukemia 13:14341440

32. Munker R, Midis G, Owen-Schaub L, Andreff M 1996 Soluble FAS (CD95) is not elevated in the serum of patients with myeloid leukemias, myeloproliferative and myelodysplastic syndromes. Leukemia 10:1531-1533

33. Hori Y, Wada H, Mori Y, Shimura M, Hiyoyama K, Nakasaki T, Nishiik, Tamaki S, Nishikawa M, Deguchi K, Minami N, Shiku H 1999 Plasma sFas and sFas ligand levels in patients with thrombotic thrombocytopenic purpura and in those with disseminated intravascular coagulation. Am J Hematol 61:21-25

34. Al-Maini M, Mountz J, Al-Mohri H, El-Ageb E, Al-Riyami B, Svenson K, Zhou T, Richens E 2000 Serum levels of soluble Fas correlate with indices of organ damage in systemic lupus erythematosus. Lupus 9:132-139

35. Iio S, Hayashi N, Mita E, Ueda K, Mochizuki K, Hiramatsu N, Kanto T, Sasaki Y, Kasahara A, Hori M 1998 Serum levels of soluble Fas antigen in chronic hepatitis C patients. J Hepatol 29:517-523

36. Rodriguez I, Matsuura K, Ody C, Nagata S, Vassalli P 1996 Systemic injection of a tripeptide inhibits the intercellular activation of CPP32-like proteases in vivo and fully protects mice against Fas-mediated fulminant liver destruction and death. J Exp Med 184:2067-2072

37. Rodriquez I, Matsuura K, Khatib K, Reed JC, Nagata S, Vassalli P 1996 A bcl-2 transgene expressed in hepatocytes protects mice from fulminant liver destruction but not from rapid death induced by anti-Fas antibody injection. J Exp Med 183:10311036

38. Lacronique V, Mignon A, Fabre M, Viollet B, Rouquet N, Molina T, Porteau A Henrion A, Bluscary D, Varlet P, Joulin V, Kahn A 1996 Bcl-2 protects from lethal hepatic apoptosis induced by an anti-Fas antibody in mice. Nat Med 2:80-86

39. Kosai K, Matsumoto K, Nagata S, Tsujimoto Y, Nakamura T 1998 Abrogation of Fas-induced fulminant hepatic failure in mice by hepatocyte growth factor. Biochem Biophys Res Commun 244:683-690

40. Chung CS, Xu YX, Wang W, Chaudry IH, Ayala A 1998 Is Fas ligand or endotoxin responsible for mucosal lymphocyte apoptosis in sepsis? Arch Surg 133:1213-1220

41. Hattori K, Hirano T, Ushiyama C, Miyajima H, Yamakawa N, Ebata T, Wada Y, Ikeda S, Yoshino K, Tateno M, Oshimi K, Kayagaki N, Yagita H, Okumura K 1997 A metalloproteinase inhibitor prevents lethal acute graft versus host disease in mice. Blood 90:542-548

42. Hasegawa D, Kojima S, Tatsumi E, Hayakawa A, Kosaka Y, Nakamura H, Sako M, Osugi Y, Nagata S, Sano K 1998 Elevation of serum Fas ligand in patients with hemophagocytic syndrome and Diamond-Blackfan anemia. Blood 91:2793-2799 